\title{
ON CURVES AND SURFACES OF AW(k) TYPE
}

\author{
Bengü KILIÇ \\ Balikesir University \\ Faculty of Art and Sciences \\ Department of Mathematics \\ Balikesir, TURKEY \\ E-mail:benguk@balikesir.edu.tr
}

\author{
Kadri ARSLAN \\ Uludag University \\ Faculty of Art and Sciences \\ Department of Mathematics \\ Bursa, TURKEY \\ E-mail:arslan@uludag.edu.tr
}

\begin{abstract}
In the present study we consider curves and surfaces of $A W(k)(k=1,2$ or 3$)$ type. We also give related examples of curves and surfaces satisfying $A W(k)$ type conditions.

Keywords: Frenet curve, curves and surfaces of $A W(k)$ type.

\section{ÖZET}

Bu çalışmada, $A W(k)$ ( $k=1,2$ yada 3 ) tipindeki eğri ve yüzeyler gözönüne alındı. $A W(k)$ şartını sağlayan eğri ve yüzeylere örnekler verildi.
\end{abstract}

Anahtar Kelimeler: Frenet eğrisi, $A W(k)$ tipinde eğri ve yüzey.

\section{1- INTRODUCTION}

Let $f: M \rightarrow \tilde{M}$ be an isometric immersion of an $n$-dimensional connected Riemannian manifold $M$ into an $m$-dimensional Riemannian manifold $\widetilde{M}$. Letters $X, Y$ and $Z$ (resp. $\zeta, \mu$ and $\xi$ ) vector fields tangent (resp. normal) to $M$. We denote the tangent bundle of $M(\operatorname{resp} . \tilde{M})$ by $T M$ (resp. $T \widetilde{M})$, unit tangent bundle of $M$ by $U M$ and the normal bundle by $T^{\perp} M$. Let $\widetilde{\nabla}$ and $\nabla$ be the Levi-Civita connections of $\tilde{M}$ and $M$, resp. Then the Gauss formula is given by

$$
\widetilde{\nabla}_{X} Y=\nabla_{X} Y+h(X, Y)
$$

where $h$ denotes the second fundamental form. The Weingarten formula is given by

$$
\widetilde{\nabla}_{X} \xi=-A_{\xi} X+D_{X} \xi
$$

where $A$ denotes the shape operator and $D$ the normal connection. Clearly $h(X, Y)=$ $h(Y, X)$ and $A$ is related to $h$ as $\left\langle A_{\xi} X, Y\right\rangle=\langle h(X, Y), \xi\rangle$, where $\langle$,$\rangle denotes the$ Riemannian metrics of $M$ and $\tilde{M}$ [1].

Let $\left\{e_{1}, e_{2}, \ldots, e_{n}, e_{n+1}, \ldots, e_{m}\right\}$ be an local orthonormal frame field on $M$ where $\left\{e_{1}, e_{2}, \ldots, e_{n}\right\}$ are tangent vector and $\left\{e_{n+1}, \ldots, e_{m}\right\}$ are normal vector. The connection form $w_{i}^{j}$ are defined by

$$
\begin{aligned}
& \widetilde{\nabla}_{e_{i}}=\sum w_{i}^{j} e_{j} ; w_{i}^{j}=-w_{j}^{i}, \quad 1 \leq i, j \leq m \\
& \nabla_{e_{i}} e_{j}=\sum_{k=1}^{n} w_{j}^{k}\left(e_{i}\right) e_{k}, \\
& D_{e_{i}} e_{\alpha}=\sum_{\beta=n+1}^{m} w_{\alpha}^{\beta}\left(e_{i}\right) e_{\beta} .
\end{aligned}
$$

The covariant derivations of $h$ is defined by 


$$
\left(\bar{\nabla}_{X} h\right)(Y, Z)=D_{X} h(Y, Z)-h\left(\nabla_{X} Y, Z\right)-h\left(Y, \nabla_{X} Z\right),
$$

where $X, Y, Z$ tangent vector fields over $M$ and $\bar{\nabla}$ is the van der Waerden Bortolotti connection. Then we have

$$
\left(\bar{\nabla}_{X} h\right)(Y, Z)=\left(\bar{\nabla}_{Y} h\right)(X, Z)=\left(\bar{\nabla}_{Z} h\right)(Y, X)
$$

which is called codazzi equations.

If $\bar{\nabla} h=0$ then $M$ is said to have parallel second fundamental form (i.e.1parallel ) [2].

It is well known that $\bar{\nabla} h$ is a normal bundle valued tensor of type $(0,3)$. We define the second covariant derivative of $h$ by

$$
\begin{aligned}
\left(\bar{\nabla}_{W} \bar{\nabla}_{X} h\right)(Y, Z)= & D_{W}\left(\left(\bar{\nabla}_{X} h\right)(Y, Z)\right)-\left(\bar{\nabla}_{X} h\right)\left(\nabla_{W} Y, Z\right)- \\
& -\left(\bar{\nabla}_{X} h\right)\left(Y, \nabla_{W} Z\right)-\left(\bar{\nabla}_{\nabla_{W} X} h\right)(Y, Z)
\end{aligned}
$$

For the orthonormal frame $\left\{e_{1}, e_{2}, \ldots, e_{n}\right\}$ of $T_{p} M$ the mean curvature vector $H$ of $f$ is defined by

$$
H=\frac{1}{n} \sum_{i=1}^{n} h\left(e_{i}, e_{i}\right)
$$

\section{CURVES OF $A W(k)$ TYPE}

Let $\gamma=\gamma(s): I \subset I E \rightarrow I E^{m}$ be a unit speed curve in $I E^{m}$. The curve $\gamma$ is called Frenet curve of osculating order $d$ if its higher order derivatives $\gamma^{\prime}(s), \gamma^{\prime \prime}(s)$, $\gamma^{\prime \prime \prime}(s), \ldots, \gamma^{(d)}(s)$ are linearly independent and $\gamma^{\prime}(s), \gamma^{\prime \prime}(s), \gamma^{\prime \prime \prime}(s), \ldots, \gamma^{(d+1)}(s)$ are linearly dependent for all $s \in I$. For each Frenet curve of order d one can associate an orthonormal $d$-frame $v_{1}, v_{2}, \ldots, v_{\mathrm{d}}$ along $\gamma$ ( such that $T=\gamma^{\prime}(s)=v_{1}$ ) called the Frenet frame and $d-1$ functions $\kappa_{1}, \kappa_{2}, \ldots, \kappa_{d-1}: I \rightarrow I R$ called the Frenet curvatures, such that the Frenet formulas are defined in the usual way;

$$
\begin{aligned}
& T^{\prime}(s)=v_{1}{ }^{\prime}=\kappa_{1}(s) v_{2}(s) \\
& v_{2}{ }^{\prime}(s)=-\kappa_{1}(s) T(s)+\kappa_{2}(s) v_{3}(s) \\
& v_{i}{ }^{\prime}(s)=-\kappa_{i-1}(s) v_{i-1}(s)+\kappa_{i}(s) v_{i+1}(s) \\
& v_{i+1}{ }^{\prime}(s)=-\kappa_{i}(s) v_{i}(s) .
\end{aligned}
$$

A regular curve $\gamma=\gamma(s): I \subset I E \rightarrow I E^{m}$ is called a $W$-curve of rank $d$, if $\gamma$ is a Frenet curve of osculating order $\mathrm{d}$ and the Frenet curvatures $\kappa_{i}, 1 \leq i \leq d-1$ are non zero constant and $\kappa_{d}=0$. In particular, a W-curve $\gamma(s)$ of rank 2 is called a geodesic circle. A W-curves of rank 3 is a right circular helix.

Let $M$ be a smooth $n$-dimensional submanifold in $(n+d)$-dimensional Euclidean space $I E^{n+d}$. For $x \in M$ and a unit vector $X \in T_{X} M$, the vector $X$ and the normal space $N_{\mathrm{x}} M$ determine a $(d+1)$-dimensional affine subspace $\operatorname{IE}(x, X)$ of $I E^{n+d}$. The intersection of $M$ and $\operatorname{IE}(x, X)$ gives rise to a curve $\gamma(s)$ (in a neighborhood of $x$ ) called the normal section of $M$ at $x$ in the direction of $X$, where $s$ denotes the arc length of $\gamma$ [1].

Definition 1. If each normal section $\gamma$ of $M$ is a Frenet curve of osculating order $\mathrm{d}$ then $M$ is said to have $d$-planar normal sections ( $d-P N S$ ). For every normal sections $\gamma$ of $M$ if $\gamma$ is a $W$-curve of rank $\mathrm{d}$ in $M$ then $M$ is called weak helical submanifold of order $d$. 
Definition 2. If each d-planar normal section is $\gamma$ a geodesic of $M$ then $M$ is said to have geodesic d-planar normal sections (Gd-PNS). For every geodesic normal sections $\gamma$ of $M$ if $\gamma$ is a $W$-curve of rank $\mathrm{d}$ in $M$ then $M$ is called weak geodesic helical submanifold of order $d$.

From now on we consider the Frenet curve of osculating order 3 of $I E^{m}$.

Proposition 3. Let $\gamma$ be a Frenet curve of $I E^{m}$ of osculating order 3 then we have

$$
\begin{gathered}
\gamma^{\prime \prime}(s)=\kappa_{1} v_{2}, \quad \gamma^{\prime}(s)=v_{1}(s) \\
\gamma^{\prime \prime \prime}(s)=-\kappa_{1}^{2} \nu_{1}+\kappa_{1}{ }^{\prime} \nu_{2}+\kappa_{1} \kappa_{2} v_{3} \\
\gamma^{\prime v}(s)=-3 \kappa_{1} \kappa_{1}{ }^{\prime} v_{1}+\left(-\kappa_{1}^{3}+\kappa_{1}{ }^{\prime \prime}-\kappa_{1} \kappa_{2}^{2}\right) \nu_{2}+\left(2 \kappa_{1}{ }^{\prime} \kappa_{2}+\kappa_{1} \kappa_{2}{ }^{\prime}\right) \nu_{3} .
\end{gathered}
$$

Notation: Let us write

$$
\begin{aligned}
& N_{1}(s)=\kappa_{1} v_{2} \\
& N_{2}(s)=\kappa_{1}{ }^{\prime} v_{2}+\kappa_{1} \kappa_{2} v_{3} \\
& N_{3}(s)=\left(-\kappa_{1}^{3}+\kappa_{1}{ }^{\prime \prime}-\kappa_{1} \kappa_{2}^{2}\right) v_{2}+\left(2 \kappa_{1}{ }^{\prime} \kappa_{2}+\kappa_{1} \kappa_{2}{ }^{\prime}\right) v_{3} .
\end{aligned}
$$

Corollary 4. $\gamma^{\prime}(s), \gamma^{\prime \prime}(s), \gamma^{\prime \prime \prime}(s)$ and $\gamma^{\prime v}(s)$ are linearly dependent if and only if $N_{1}(\mathrm{~s}), N_{2}(s)$ and $N_{3}(s)$ are linearly dependent.

Theorem 5. Let $\gamma$ be a Frenet curve of $I E^{m}$ of osculating order 3 then

$$
N_{3}(s)=\left\langle N_{3}(s), N_{1}^{*}(s)\right\rangle N_{1}^{*}(s)+\left\langle N_{3}(s), N_{2}^{*}(s)\right\rangle N_{2}^{*}(s)
$$

where

$$
N_{1}^{*}(s)=\frac{N_{1}(s)}{\left\|N_{1}(s)\right\|}, \quad N_{2}^{*}(s)=\frac{N_{2}(s)-\left\langle N_{2}(s), N_{1}^{*}(s)\right\rangle N_{1}^{*}(s)}{\left\|N_{2}(s)-\left\langle N_{2}(s), N_{1}^{*}(s)\right\rangle N_{1}^{*}(s)\right\|}
$$

[3].

Definition 6. Frenet curves ( of osculating order 3 ) are

i)of type weak $A W(2)$ if they satisfy

$$
N_{3}(s)=\left\langle N_{3}(s), N_{2}^{*}(s)\right\rangle N_{2}^{*}(s),
$$

ii) of type weak $A W(3)$ if they satisfy

$$
N_{3}(s)=\left\langle N_{3}(s), N_{1}^{*}(s)\right\rangle N_{1}^{*}(s)
$$

Corollary 7. Let $\gamma$ be a Frenet curve of type weak $A W(2)$. If $\gamma$ is a plane curve then $\kappa_{1}{ }^{\prime \prime}(s)-\kappa_{1}^{3}(s)=0$, and the solution of this differential equation is

$$
\kappa_{1}= \pm \frac{\sqrt{2}}{s+c}, \quad c=\text { Const. }
$$

The curvature vector field of $\gamma$ ( the mean curvature vector field of $\gamma$ ) is defined by

$$
h(T, T)=H(s)=\gamma^{\prime \prime}(s)=\kappa_{1}(s) v_{2}(s) .
$$

One can use the Frenet equations (15) to compute 


$$
\gamma^{\prime \nu \perp}(s)=\left(-\kappa_{1}^{3}+\kappa_{1}{ }^{\prime \prime}-\kappa_{1} \kappa_{2}^{2}\right) \nu_{2}+\left(2 \kappa_{1}{ }^{\prime} \kappa_{2}+\kappa_{1} \kappa_{2}{ }^{\prime}\right) \nu_{3}
$$

Definition 8.Curves are of type $A W(1)$ if they satisfy

$$
\gamma^{\prime v \perp}(s)=0,
$$

of type $A W(2)$ if they satisfy

$$
\gamma^{\prime \nu \perp}(s) \Lambda \gamma^{\prime \prime \prime}(s)=0
$$

and of type $A W(3)$ if they satisfy

$$
\gamma^{\prime v \perp}(s) \Lambda \gamma^{\prime \prime}(s)=0 \text {. }
$$

Proposition 9. Let $\gamma$ be a Frenet curve of type $A W(1)$ if and only if

$$
\begin{aligned}
& -\kappa_{1}^{3}+\kappa_{1}{ }^{\prime \prime}-\kappa_{1} \kappa_{2}^{2}=0 \\
& 2 \kappa_{1}{ }^{\prime} \kappa_{2}+\kappa_{1} \kappa_{2}{ }^{\prime}=0 .
\end{aligned}
$$

Proof. Substituting (17) into (18) we get the result.

Corollary 10. Let $\gamma$ be a Frenet curve of type $A W(1)$.

i)If $\kappa_{1}=0$ then $\gamma$ is a straight line.

ii)If $\kappa_{1} \neq 0, \kappa_{2}=0$ then $\kappa_{1}{ }^{\prime \prime}-\kappa_{1}^{3}=0$. That is

$$
\kappa_{1}= \pm \frac{\sqrt{2}}{s+c}, c=\text { Const. }
$$

[3].

iii)If $\kappa_{1}, \kappa_{2} \neq 0$ then by (21) and (22) we obtain

$$
\kappa_{2}=\frac{c}{\kappa_{1}^{2}}, \quad \kappa_{1}^{\prime \prime}-\kappa_{1}^{3}-\frac{c^{2}}{\kappa_{1}^{3}}=0 .
$$

Putting $\kappa_{1}=y$ into (23) we get

$$
y^{\prime \prime}-y^{3}-\frac{c^{2}}{y^{3}}=0 .
$$

Thus solving the differential equation (24) one gets

$$
\begin{gathered}
\int^{y(x)} \frac{2_{-} a}{\sqrt{2_{-} a^{6}-4 c^{2}+4{ }_{-} C 1_{-} a^{2}}} d{ }_{-} a-x-_{-} C 2=0, \\
\int^{y(x)}-\frac{2 \_a}{\sqrt{2_{-} a^{6}-4 c^{2}+4{ }_{-} C 1_{-} a^{2}}} d{ }_{-} a-x-_{-} C 2=0 .
\end{gathered}
$$

Using $\kappa_{1}=y, \kappa_{2}=\frac{c}{\kappa_{1}^{2}}$, we get the result.

Corollary 11. Every plane curve of $A W(1)$ type is also of weak $A W(2)$ type [3].

Proposition 12. Let $\gamma$ be a Frenet curve of type $A W(2)$ if and only if

$$
-\kappa_{1}^{3}+\kappa_{1}{ }^{\prime \prime}-\kappa_{1} \kappa_{2}^{2}=\delta_{1} \kappa_{1}{ }^{\prime}
$$




$$
2 \kappa_{1}{ }^{\prime} \kappa_{2}+\kappa_{1} \kappa_{2}{ }^{\prime}=\delta_{1} \kappa_{1} \kappa_{2} .
$$

Proof. Substituting (14) and (17) into (19) we get the result.

Corollary 13. Let $\gamma$ be a Frenet curve of type $A W(2)$.

i)If $\kappa_{1}=0$ then $\gamma$ is a straight line.

ii)If $\kappa_{1} \neq 0, \kappa_{2}=0$ then by (25) we obtain

$$
\kappa_{1}^{\prime \prime}-\kappa_{1}^{3}-\delta_{1} \kappa_{1}^{\prime}=0
$$

Putting $\kappa_{1}=y$ into (27) we get

$$
y^{\prime \prime}-y^{3}-\delta_{1} y^{\prime}=0
$$

Thus solving the differential equation (28) one gets

$$
y=c_{1} e^{\frac{\delta_{1}+\sqrt{4+\delta_{1}^{2}}}{2} x}+c_{2} e^{\frac{\delta_{1}-\sqrt{4+\delta_{1}^{2}}}{2} x} .
$$

Using $\kappa_{1}=y$ we get the result.

iii)If $\kappa_{1}, \kappa_{2} \neq 0$ then by (25) and (26) we obtain

$$
\kappa_{1}^{\prime \prime \prime} \kappa_{1}+\kappa_{1}^{\prime \prime}\left(3 \kappa_{1}{ }^{\prime}-3 \delta_{1} \kappa_{1}\right)+\kappa_{1}{ }^{\prime}\left(-3 \delta_{1} \kappa_{1}{ }^{\prime}-6 \kappa_{1}^{3}+2 \delta_{1}^{2} \kappa_{1}\right)+2 \delta_{1} \kappa_{1}^{4}=0
$$

Putting $\kappa_{1}=y$ and $\delta_{1}=c$ into (29) we get

$$
y^{\prime \prime \prime} y+y^{\prime \prime}\left(3 y^{\prime}-3 c y\right)+y^{\prime}\left(-3 c y^{\prime}-6 y^{3}+2 c^{2} y\right)+2 c y^{4}=0
$$

Thus solving the differential equation (30) one gets

$$
\begin{aligned}
& y(x)=0, y(x)=_{-} b\left(_{-} a\right) \text { where }\left[\left\{-_{-} b\left(_{-} a\right)^{6} e^{\left(-2 c_{-} a\right)}-_{-} b\left(\__{-} a\right)^{3} e^{\left(-2 c_{-} a\right)} c\left(\frac{d}{d_{-} a}{ }_{-} b\left({ }_{-} a\right)\right)\right.\right. \\
& \left.+_{-} b\left({ }_{-} a\right)^{3} e^{\left(-2 c_{-} a\right)}\left(\frac{d^{2}}{d_{-} a^{2}}{ }_{-} b\left({ }_{-} a\right)\right)++_{-} C 1=0\right\}, \\
& \left.\left\{\_a=x, \__{-} b\left(\_a\right)=y(x)\right\}, \quad\left\{x={ }_{-} a, y(x)=_{-} b\left({ }_{-} a\right)\right\}\right] .
\end{aligned}
$$

Using $\kappa_{1}=y$ we get the result.

Proposition 14. Let $\gamma$ be a Frenet curve of type $A W(3)$ if and only if

$$
\begin{aligned}
& -\kappa_{1}^{3}+\kappa_{1}{ }^{\prime \prime}-\kappa_{1} \kappa_{2}^{2}=\delta_{2} \kappa_{1} \\
& 2 \kappa_{1}{ }^{\prime} \kappa_{2}+\kappa_{1} \kappa_{2}{ }^{\prime}=0 .
\end{aligned}
$$

Proof. Substituting (16) and (17) into (20) we get the result.

Corollary 15. Let $\gamma$ be a Frenet curve of type $A W(3)$.

i)If $\kappa_{1}=0$ then $\gamma$ is a straight line.

ii)If $\kappa_{1} \neq 0, \kappa_{2}=0$ then by (31) we obtain

$$
\kappa_{1}^{\prime \prime}-\kappa_{1}^{3}-\delta_{2} \kappa_{1}=0
$$

Putting $\kappa_{1}=y$ and $\delta_{2}=c$ into (33) we get

$$
y^{\prime \prime}-y^{3}-c y=0
$$

Thus solving the differential equation (34) one gets

$$
\int^{y(x)} \frac{2}{\sqrt{2 a^{4}+4 a_{-} a^{2} c+4 \_C 1}} d \_a-x-_{-} C 2=0,
$$




$$
\int^{y(x)}-\frac{2}{\sqrt{2_{-} a^{4}+4_{-} a^{2} c+4_{-} C 1}} d \_a-x-_{-} C 2=0 \text {. }
$$

Using $\kappa_{1}=y$ we get the result.

iii)If $\kappa_{1}, \kappa_{2} \neq 0$ then by (31) and (32) we obtain

$$
\kappa_{2}=\frac{c}{\kappa_{1}^{2}}, \quad \kappa_{1}^{\prime \prime}-\kappa_{1}^{3}-\frac{c^{2}}{\kappa_{1}^{3}}-\delta_{2} \kappa_{1}=0 .
$$

Putting $\kappa_{1}=y$ and $\delta_{2}=d$ into (35) we get

$$
y^{\prime \prime}-y^{3}-\frac{c^{2}}{y^{3}}-d y=0 \text {. }
$$

Thus solving the differential equation (36) one gets

$$
\begin{aligned}
& \int^{y(x)}-\frac{2{ }_{-} a}{\sqrt{4_{-} C 1_{-} a^{2}+2 a_{-} a^{6}-4 c^{2}+4 d_{-} a^{4}}} d_{-} a-x-_{-} C 2=0 \\
& \int^{y(x)} \frac{2{ }_{-} a}{\sqrt{4_{-} C 1_{-} a^{2}+2 a_{-} a^{6}-4 c^{2}+4 d_{-} a^{4}}} d_{-} a-x-_{-} C 2=0 .
\end{aligned}
$$

Using $\kappa_{1}=y, \kappa_{2}=\frac{c}{\kappa_{1}^{2}}$, we get the result.

Corollary 16. Every Frenet curve of weak $A W(3)$ type is also of $A W(3)$ type [3].

\section{SURFACES OF AW(k) TYPE}

In this part we consider surfaces of $A W(k)$ type.

Let us write

$$
\begin{aligned}
& H(X)=h(X, X) \\
& \nabla H(X)=\left(\bar{\nabla}_{X} h\right)(X, X) \\
& J(X)=\left(\bar{\nabla}_{X} \bar{\nabla}_{X} h\right)(X, X)+3 h\left(A_{h(X, X)} X, X\right)
\end{aligned}
$$

so that $H: T(M) \rightarrow N(M), \nabla H: T(M) \rightarrow N(M)$ and $J: T(M) \rightarrow N(M)$ are fibre maps whose restriction to each fibre $T_{X}(M)$ is a homogeneous polynomial map, $H$ is of degree 2 and $\nabla H$ is of degree 3 and $J$ is of degree 4 .

Then

$$
\begin{aligned}
& J_{1}\left(\bar{\nabla}_{e_{1}} \bar{\nabla}_{e_{1}} h\right)\left(e_{1}, e_{1}\right)+3 h\left(A_{h\left(e_{1}, e_{1}\right)} e_{1}, e_{1}\right) \\
& J_{2}\left(\bar{\nabla}_{e_{2}} \bar{\nabla}_{e_{2}} h\right)\left(e_{2}, e_{2}\right)+3 h\left(A_{h\left(e_{2}, e_{2}\right)} e_{2}, e_{2}\right) .
\end{aligned}
$$

Definition 17. [4] Submanifolds are of type $A W(1)$ if they satisfy

$$
J \equiv 0
$$

submanifolds are of type $A W(2)$ if they satisfy

$$
\|\nabla H\|^{2} J \equiv\langle J, \nabla H\rangle \nabla H
$$

and of type $A W(3)$ if they satisfy

$$
\|H\|^{2} J \equiv\langle J, H\rangle H \text {. }
$$


Proposition 18. [5] Let $M$ be a connected normally flat surfaces in $I E^{4} . e_{3}$ is parallel to the mean curvature vector $H$ of $M$ such that

$$
A_{e_{3}}=\left[\begin{array}{cc}
\lambda & 0 \\
0 & \mu
\end{array}\right], \quad A_{e_{4}}=\left[\begin{array}{cc}
\beta & 0 \\
0 & -\beta
\end{array}\right] \text {. }
$$

We give the following results.

Lemma 19. From the Codazzi equations and using (4), (5) and (45)

$$
\begin{aligned}
& (\lambda-\mu) w_{1}^{2}\left(e_{2}\right)=e_{1}(\mu)+\beta w_{3}^{4}\left(e_{1}\right) \\
& 2 \beta w_{1}^{2}\left(e_{2}\right)=-e_{1}(\beta)+\mu w_{3}^{4}\left(e_{1}\right) \\
& (\lambda-\mu) w_{1}^{2}\left(e_{1}\right)=e_{2}(\lambda)-\beta w_{3}^{4}\left(e_{2}\right) \\
& 2 \beta w_{1}^{2}\left(e_{1}\right)=e_{2}(\beta)+\lambda w_{3}^{4}\left(e_{2}\right) .
\end{aligned}
$$

Lemma 20. If $M \subset I E^{4}$ is normally flat surfaces then

$$
\begin{gathered}
J_{1}=\left\{e_{1}^{2}(\lambda)-\lambda\left(w_{3}^{4}\left(e_{1}\right)\right)^{2}-2 e_{1}(\beta) w_{3}^{4}\left(e_{1}\right)-\beta e_{1}\left(w_{3}^{4}\left(e_{1}\right)\right)-3 w_{1}^{2}\left(e_{1}\right) e_{2}(\lambda)(50)\right. \\
\left.+3 \beta w_{1}^{2}\left(e_{1}\right) w_{3}^{4}\left(e_{2}\right)+3 \lambda\left(\lambda^{2}+\beta^{2}\right)\right\} e_{3} \\
+\left\{e_{1}^{2}(\beta)-\beta\left(w_{3}^{4}\left(e_{1}\right)\right)^{2}+2 e_{1}(\lambda) w_{3}^{4}\left(e_{1}\right)+\lambda e_{1}\left(w_{3}^{4}\left(e_{1}\right)\right)-3 w_{1}^{2}\left(e_{1}\right) e_{2}(\beta)\right. \\
\left.-3 \lambda w_{1}^{2}\left(e_{1}\right) w_{3}^{4}\left(e_{2}\right)+3 \beta\left(\lambda^{2}+\beta^{2}\right)\right\} e_{4}
\end{gathered}
$$

and

$$
\begin{gathered}
J_{2}=\left\{e_{2}^{2}(\mu)-\mu\left(w_{3}^{4}\left(e_{2}\right)\right)^{2}+2 e_{2}(\beta) w_{3}^{4}\left(e_{2}\right)+\beta e_{2}\left(w_{3}^{4}\left(e_{2}\right)\right)+3 w_{1}^{2}\left(e_{2}\right) e_{1}(\mu)\right. \\
\left.+3 \beta w_{1}^{2}\left(e_{2}\right) w_{3}^{4}\left(e_{1}\right)+3 \mu\left(\mu^{2}+\beta^{2}\right)\right\} e_{3} \\
+\left\{-e_{2}^{2}(\beta)+\beta\left(w_{3}^{4}\left(e_{2}\right)\right)^{2}+2 e_{2}(\mu) w_{3}^{4}\left(e_{2}\right)+\mu e_{2}\left(w_{3}^{4}\left(e_{2}\right)\right)-3 w_{1}^{2}\left(e_{2}\right) e_{1}(\beta)\right. \\
\left.+3 \mu w_{1}^{2}\left(e_{2}\right) w_{3}^{4}\left(e_{1}\right)-3 \beta\left(\mu^{2}+\beta^{2}\right)\right\} e_{4} .
\end{gathered}
$$

Proposition 21. Let $M \subset I E^{4}$ be a normally flat surfaces. If $M$ is $A W(1)$ type then $J_{1}=0$ and $J_{2}=0$. That is

$$
\begin{gathered}
e_{1}^{2}(\lambda)-(\lambda+2 \mu)\left(w_{3}^{4}\left(e_{1}\right)\right)^{2}+4 \beta w_{3}^{4}\left(e_{1}\right) w_{1}^{2}\left(e_{2}\right)-\beta e_{1}\left(w_{3}^{4}\left(e_{1}\right)\right) \\
-3(\lambda-\mu)\left(w_{1}^{2}\left(e_{1}\right)\right)^{2}+3 \lambda\left(\lambda^{2}+\beta^{2}\right)=0, \\
2 e_{1}(\lambda) w_{3}^{4}\left(e_{1}\right)+(\lambda+\mu) e_{1}\left(w_{3}^{4}\left(e_{1}\right)\right)+(\lambda-3 \mu) w_{3}^{4}\left(e_{1}\right) w_{1}^{2}\left(e_{2}\right) \\
-\beta\left\{2\left(w_{3}^{4}\left(e_{1}\right)\right)^{2}-4\left(w_{1}^{2}\left(e_{2}\right)\right)^{2}+2 e_{1}\left(w_{1}^{2}\left(e_{2}\right)\right)+6\left(w_{1}^{2}\left(e_{1}\right)\right)^{2}-3\left(\lambda^{2}+\beta^{2}\right)\right\}=0, \\
e_{2}^{2}(\mu)-(\mu+2 \lambda)\left(w_{3}^{4}\left(e_{2}\right)\right)^{2}+4 \beta w_{3}^{4}\left(e_{2}\right) w_{1}^{2}\left(e_{1}\right)+\beta e_{2}\left(w_{3}^{4}\left(e_{2}\right)\right) \\
+3(\lambda-\mu)\left(w_{1}^{2}\left(e_{2}\right)\right)^{2}+3 \mu\left(\mu^{2}+\beta^{2}\right)=0,
\end{gathered}
$$

$$
\begin{gathered}
2 e_{2}(\mu) w_{3}^{4}\left(e_{2}\right)+(\lambda+\mu) e_{2}\left(w_{3}^{4}\left(e_{2}\right)\right)+(3 \lambda-\mu) w_{3}^{4}\left(e_{2}\right) w_{1}^{2}\left(e_{1}\right) \\
-\beta\left\{-2\left(w_{3}^{4}\left(e_{2}\right)\right)^{2}+4\left(w_{1}^{2}\left(e_{1}\right)\right)^{2}+2 e_{2}\left(w_{1}^{2}\left(e_{1}\right)\right)-6\left(w_{1}^{2}\left(e_{2}\right)\right)^{2}+3\left(\mu^{2}+\beta^{2}\right)\right\}=0 .
\end{gathered}
$$


Proof. Substituting (46), (47), (48), (49) into (50) and (51) and from $A W(1)$ type definition we get the result.

Proposition 22.Let $M$ be a normally flat and has got constant principal curvature submanifold. Then

$$
\begin{aligned}
J_{1}=\{ & \left.-\lambda\left(w_{3}^{4}\left(e_{1}\right)\right)^{2}-\beta e_{1}\left(w_{3}^{4}\left(e_{1}\right)\right)+3 \beta w_{1}^{2}\left(e_{1}\right) w_{3}^{4}\left(e_{2}\right)+3 \lambda\left(\lambda^{2}+\beta^{2}\right)\right\} e_{3} \\
& +\left\{-\beta\left(w_{3}^{4}\left(e_{1}\right)\right)^{2}+\lambda e_{1}\left(w_{3}^{4}\left(e_{1}\right)\right)-3 \lambda w_{1}^{2}\left(e_{1}\right) w_{3}^{4}\left(e_{2}\right)+3 \beta\left(\lambda^{2}+\beta^{2}\right)\right\} e_{4}, \\
J_{2}=\{ & \left.-\lambda\left(w_{3}^{4}\left(e_{2}\right)\right)^{2}+\beta e_{2}\left(w_{3}^{4}\left(e_{2}\right)\right)+3 \beta w_{1}^{2}\left(e_{2}\right) w_{3}^{4}\left(e_{1}\right)+3 \lambda\left(\lambda^{2}+\beta^{2}\right)\right\} e_{3} \\
& +\left\{\beta\left(w_{3}^{4}\left(e_{2}\right)\right)^{2}+\lambda e_{2}\left(w_{3}^{4}\left(e_{2}\right)\right)+3 \lambda w_{1}^{2}\left(e_{2}\right) w_{3}^{4}\left(e_{1}\right)-3 \beta\left(\lambda^{2}+\beta^{2}\right)\right\} e_{4} .
\end{aligned}
$$

Lemma 23. Let $M$ be a normally flat and has got constant principal curvature submanifold of $A W(1)$ type

i) If $\lambda=\beta=0$ then $M$ is a plane,

ii) If $\lambda=-\beta$ then $M$ has got vanishing Gaussian curvature $(K=0)$, mean curvature $H=\lambda$ or $\left(w_{3}^{4}\left(e_{2}\right)\right)^{2}=3\left(\lambda^{2}+\beta^{2}\right)$,

iii) If $\lambda=\beta$ then $M$ has got vanishing Gaussian curvature $(K=0)$, mean curvature $H=$ $\lambda$ or $e_{2}\left(w_{3}^{4}\left(e_{2}\right)\right)=-3 w_{1}^{2}\left(e_{2}\right) w_{3}^{4}\left(e_{1}\right)$.

Theorem 24. [3] Let $\gamma$ be a Frenet curve of order 3 and of type $A W(k)$ then the cylinder over $\gamma$ is of type $A W(k)$, where $k=1,2,3$.

Example 25. Let $\gamma(s)=\left(\int_{0}^{s} \cos \left(P_{k}(t)\right) d t, \int_{0}^{s} \sin \left(P_{k}(t)\right) d t\right)$ be a polinomial spiral with $\kappa_{\gamma}(s)=P_{k}^{\prime}(t)= \pm \frac{\sqrt{2}}{s+c}, \quad c=$ Const. The Riemannian product of $\gamma(s)$ with the helicoid $x(w, t)=($ wcost $, w \sin t, a t)$ is of $A W(1)$ type.

Example 26. We define helical cylinder $\mathbf{H}^{2}$ embedded in $I E^{4}$ by

$$
x(u, v)=\{(u, a \cos v, a \sin v, b v): a, b \in I R\}
$$

and we show that $\mathbf{H}^{2}$ is of type $A W(3)$.

For

$T_{p}\left(\mathbf{H}^{2}\right)$ is spanned by

$$
\mathrm{p}=(u, a \cos v, a \sin v, b v)
$$

$$
\begin{aligned}
& x_{u}=(1,0,0,0) \\
& x_{v}=(0,-a \sin v, a \cos v, b)
\end{aligned}
$$

and $N_{p}\left(\mathbf{H}^{2}\right)$ is spanned by

$$
\begin{aligned}
& n_{1}=(0, \cos v, \sin v, 0) \\
& n_{2}=\left(0, \frac{b}{a} \sin v,-\frac{b}{a} \cos v, 1\right) .
\end{aligned}
$$

We have the orthonormal frame $X, Y, v_{1}, v_{2}$ where

$$
X=\frac{X_{u}}{\left\|x_{u}\right\|}=(1,0,0,0)
$$


Differentiating these we have

$$
\begin{aligned}
& Y=\frac{X_{v}}{\left\|x_{v}\right\|}=\frac{1}{\sqrt{a^{2}+b^{2}}}(0,-a \sin v, a \cos v, b) \\
& v_{1}=\frac{n_{1}}{\left\|n_{1}\right\|}=(0, \cos v, \sin v, 0) \\
& v_{2}=\frac{n_{2}}{\left\|n_{2}\right\|}=\frac{a}{\sqrt{a^{2}+b^{2}}}\left(0, \frac{b}{a} \sin v,-\frac{b}{a} \cos v, 1\right) .
\end{aligned}
$$

$$
\begin{gathered}
\tilde{\nabla}_{X} X=\widetilde{\nabla}_{X} Y=\widetilde{\nabla}_{Y} X=0, \quad \widetilde{\nabla}_{Y} Y=\frac{-a}{a^{2}+b^{2}} v_{1} \\
\widetilde{\nabla}_{X} v_{1}=\widetilde{\nabla}_{X} v_{2}=0, \quad \widetilde{\nabla}_{Y} v_{1}=\frac{a}{a^{2}+b^{2}} Y-\frac{b}{a^{2}+b^{2}} v_{2}, \quad \widetilde{\nabla}_{Y} v_{2}=\frac{b}{a^{2}+b^{2}} v_{1} .
\end{gathered}
$$

Combining these with (1) and (2) we get

$$
\begin{aligned}
& \nabla_{X} X=\nabla_{X} Y=\nabla_{Y} X=\nabla_{Y} Y=0 \\
& h(X, X)=h(X, Y)=h(Y, X)=0, \quad h(Y, Y)=\frac{-a}{a^{2}+b^{2}} v_{1} \\
& A_{v_{1}} X=A_{v_{2}} X=A_{v_{2}} Y=0, \quad A_{v_{1}} Y=\frac{-a}{a^{2}+b^{2}} Y \\
& D_{X} v_{1}=D_{X} v_{2}=0, \quad D_{Y} v_{1}=\frac{-b}{a^{2}+b^{2}} v_{2}, \quad D_{Y} v_{2}=\frac{b}{a^{2}+b^{2}} v_{1}
\end{aligned}
$$

Substituting (6), (8), (54), (55), (56) and (57) into (40) and (41) we have

$$
J(X)=J_{1}=0, \quad J(Y)=J_{2}=\frac{a\left(b^{2}-3 a^{2}\right)}{\left(a^{2}+b^{2}\right)^{3}} v_{1} .
$$

Substituting (37) and (58) into (44) we get the result.

Example 27. We define surfaces embedded in $I E^{4}$ by $x(u, v)=(u, v, u \cos v, u \sin v)$

and we show that surfaces is of type $A W(3)$.

After some calculations we get

$$
J(X)=J_{1}=0, \quad J(Y)=J_{2}=\frac{-\sqrt{2} u}{\left(1+u^{2}\right)^{3}} v_{1} .
$$

Substituting (37) and (59) into (44) we get the result.

Example 28. We define surfaces embedded in $\mathrm{IE}^{4}$ by

$$
x(u, v)=\{(u \cos v, u \sin v, \cos b v, \sin b v): b \in I R\}
$$

and we show that surfaces is of type $A W(3)$.

After some calculations we get

$$
J(X)=J_{1}=0, \quad J(Y)=J_{2}=\frac{b^{2}\left(u^{2} b^{2}+8 u^{2}-3 b^{4}\right)}{\left(u^{2}+b^{2}\right)^{3}} v_{1} .
$$

Substituting (37) and (60) into (44) we get the result.

Example 29. We define a Mobius band $\mathbf{M}^{2}$ embedded in $I E^{4}$ by

$$
x(u, v)=\left(\cos u, \sin u, v \cos \frac{u}{2}, v \sin \frac{u}{2}\right)
$$


and we show that $\mathbf{M}^{2}$ is of type $A W(3)$.

After some calculations we get

$$
J(X)=J_{1}=\frac{-144}{\left(4+v^{2}\right)^{3}} v_{1}, \quad J(Y)=J_{2}=0 .
$$

Substituting (37) and (61) into (44) we get the result.

\section{REFERENCES}

[1] B.Y. Chen, "Geometry of Submanifolds", Dekker (1973).

[2] U. Lumiste, "Submanifolds with Parallel Fundamental Form ", Handbook of Differential Geometry (1999) Vol 1, Chapter 7, 86.

[3] K. Arslan, C. Ozgur, "Curves and Surfaces of AW(k) Type", Geometry and Topology of Submanifolds IX (Valenciennes/Lyon/Leuven, 1997) World Sci. Publishing, River Edge, NJ, (1999) 21-26.

[4] K. Arslan, "Isoparametric Submanifolds With Pointwise k-Planar Normal Sections", PhD. Thesis, The University of Leeds, (1993).

[5] J. Deprez, "Semi-parallel Surfaces in Euclidean Spaces", Journal of Geometry, (1985) Vol 25, 192-200. 